\title{
Approximation of the Integral Boundary Layer Equation by the Kuramoto-Sivashinsky Equation *
}

\author{
Hannes Uecker ${ }^{\dagger}$
}

February 10, 2002

\begin{abstract}
In suitable parameter regimes the Integral Boundary Layer equation (IBLe) can be formally derived as a long wave approximation for the flow of a viscous incompressible fluid down an inclined plane. For very long waves with small amplitude, the IBLe can be further reduced to the Kuramoto-Sivashinsky equation (KSe). Here we justify this reduction of the IBL to the KSe. Using energy estimates we show that solutions of the KSe approximate solutions of the IBLe over sufficiently long time scales. This is a step towards understanding the approximation properties of the KSe for the full Navier-Stokes system describing the inclined film flow.
\end{abstract}

\section{Introduction}

For typical flow conditions the so called Nusselt flow of a viscous incompressible fluid down an inclined plane is subject to long wave surface instabilities and trains of solitary waves develop on the free surface. Starting from the Navier-Stokes equations, a number of reduced equations have been formally derived to describe the evolution of the free surface and in particular to understand the formation of these wavetrains.

Here we study analytically the relation between two of the approximate equations. The first one is the so called Integral Boundary Layer equation (IBLe) which is derived from the Navier-Stokes equation using a long wave expansion followed by an averaging over the film height. In Appendix B we briefly review this derivation of the IBLe; see also [3] for an extensive review and [10] for experiments on inclined film flows.

By a small amplitude and second long wave expansion in the IBLe, corresponding to a small amplitude and very long wave expansion of the Navier-Stokes equation, the IBLe can be further reduced to the Kuramoto-Sivashinsky equation (KSe). This second reduction

*The author is grateful for support by the Deutsche Forschungsgemeinschaft under grant UE60/1. He thanks R.L. Pego for helpful discussions and remarks.

${ }^{\dagger}$ Department of Mathematics, University of Maryland, College Park, 20740 MD, USA, huecker@umd.math.edu; new address: Math. Institut I, Universität Karlsruhe, 76128 Karlsruhe, Germany 
is justified in this paper; we show that the KS-dynamics can be observed in the IBLe, see Theorem 1.1.

Using the time and space scales of the Navier-Stokes equations, the IBLe we consider reads

$$
\begin{aligned}
h_{t}= & -q_{x}, \\
q_{t}= & -\frac{6}{5} \partial_{x}\left(\frac{q^{2}}{h}\right)+\frac{2}{\mathrm{R}}\left(h-\frac{3 q}{2 h^{2}}-h_{x} h \cot \theta\right)+\mathrm{W} \varepsilon^{-2} h\left(\partial_{x}^{3} h-\frac{3}{2} \partial_{x}^{3} h h_{x}^{2}-3 h_{x x}^{2} h_{x}\right) \\
& +\frac{1}{\mathrm{R}}\left(\frac{7}{2} q_{x x}-\frac{9 q_{x} h_{x}}{h}+\frac{6 q h_{x}^{2}}{h^{2}}-\frac{9 q h_{x x}}{2 h}\right)
\end{aligned}
$$

where $x \in \mathbb{R}, t>0, h$ is the film height, $q$ describes the flow, $0<\theta \leq \pi / 2$ is the inclination angle, $\mathrm{R}$ is the Reynolds number, $\mathrm{W}$ is a normalized Weber number and $0<\varepsilon \ll 1$ is a small parameter. In the derivation of (1.1) it is assumed that the Weber number $\mathrm{W}_{e}=\mathrm{W}^{-2}=$ $\mathcal{O}\left(\varepsilon^{-2}\right)$, while $\mathrm{R}=\mathcal{O}(1)$ and $\cot \theta=\mathcal{O}(1)$. The latter means, that the plane may not be close to horizontal. However, a vertical plane, i.e. $\cot \theta=0$, is allowed. As already said, see Appendix $\mathrm{B}$ for the underlying scalings. The parameter $\mathrm{W}$ could be adsorbed into $\varepsilon$, but we think the analysis becomes more transparent by keeping $\mathrm{W}$.

In the IBLe the Nusselt solution of the inclined film problem corresponds to $(h, q)=$ $(1,2 / 3)$. Since we are interested in the instability of this solution we will assume throughout that $\mathrm{R}$ is larger than the critical Reynolds number, i.e.,

$$
\mathrm{R}>\mathrm{R}_{c}=\frac{5}{4} \cot \theta .
$$

With an abuse of notation we set $h=1+\eta, q=2 / 3+q$, and expand (1.1) up to quadratic terms, since from previous work, e.g. [8], it is well known, and it can also readily be seen in the analysis below, that cubic and higher order terms play no role in the justification of the long wave/small amplitude approximation for (1.1). See however Remarks 1.3, 3.2 and A.5 for changes in the function spaces in this case. We write this quadratic expansion as

$$
\begin{aligned}
& \eta_{t}=-q_{x} \\
& q_{t}=a_{0}(\eta) \eta+a_{1}(\eta, q) \eta_{x}+a_{2}(\eta, q) \eta_{x x}+\varepsilon^{-2} a_{3}(\eta) \eta_{x x x}-b_{0}(\eta) q-b_{1}(\eta, q) q_{x}+b_{2} q_{x x}
\end{aligned}
$$

where

$$
\begin{aligned}
& a_{0}(\eta)=(6-6 \eta) / \mathrm{R}, \quad a_{1}(\eta, q)=\left(\frac{4}{5}-\frac{2}{\mathrm{R}} \cot \theta-\frac{8}{5} \eta+\frac{8}{5} q+\frac{6}{\mathrm{R}} \eta_{x}\right) \\
& a_{2}(\eta, q)=\frac{1}{\mathrm{R}}\left(-3+\frac{9}{2} \eta-\frac{9}{2} q\right), \quad a_{3}(\eta)=\mathrm{W}(1+\eta), \\
& b_{0}(\eta)=\frac{3}{\mathrm{R}}(1-2 \eta), \quad b_{1}(\eta, q)=\frac{8}{5}-\frac{8}{5} \eta+\frac{12}{5} q+\frac{9}{\mathrm{R}} \eta_{x}, \quad b_{2}=\frac{7}{2 \mathrm{R}}
\end{aligned}
$$

Splitting (1.3) and (1.4) into linear and nonlinear terms we write

$$
\begin{gathered}
U_{t}=A_{0} U+F(U), \\
U=\left(\begin{array}{c}
\eta \\
q
\end{array}\right), \quad A_{0}=A_{0}\left(\partial_{x}\right)=\left(\begin{array}{cc}
0 & -\partial_{x} \\
a_{00}+a_{10} \partial_{x}+a_{20} \partial_{x}^{2}+\varepsilon^{-2} a_{30} \partial_{x}^{3} & -b_{00}-b_{10} \partial_{x}+b_{20} \partial_{x}^{2}
\end{array}\right),
\end{gathered}
$$


where $a_{00}=a_{0}(0), a_{10}=a_{1}(0,0), \ldots$, and where $F$ contains the quadratic terms.

Inserting $U=\mathrm{e}^{\mu t+\mathrm{i} k x} U(k)$ into (1.6) we obtain the dispersion relation

$$
\begin{aligned}
\mu_{1,2}(k)= & -\frac{1}{2}\left(\frac{7}{2 \mathrm{R}} k^{2}+\frac{8}{5} \mathrm{i} k+\frac{3}{\mathrm{R}}\right) \\
& \pm \sqrt{\frac{1}{4}\left(\frac{7}{2 \mathrm{R}} k^{2}+\frac{8}{5} \mathrm{i} k+\frac{3}{\mathrm{R}}\right)^{2}-\frac{6}{\mathrm{R}} \mathrm{i} k-\left(\frac{4}{5}-\frac{2}{\mathrm{R}} \cot \theta\right) k^{2}+\frac{3}{\mathrm{R}} \mathrm{i} k^{3}-\mathrm{W} \varepsilon^{-2} k^{4}} .
\end{aligned}
$$

This spectrum of the operator $A_{0}(\mathrm{ik})$ is sketched in figure 1 . From $\mu_{1}$ we obtain a long wave instability with maximum growth rate $\operatorname{Re} \mu_{1}\left(k_{c}\right)=\mathcal{O}\left(\varepsilon^{2}\right), k_{c}=\mathcal{O}(\varepsilon)$.
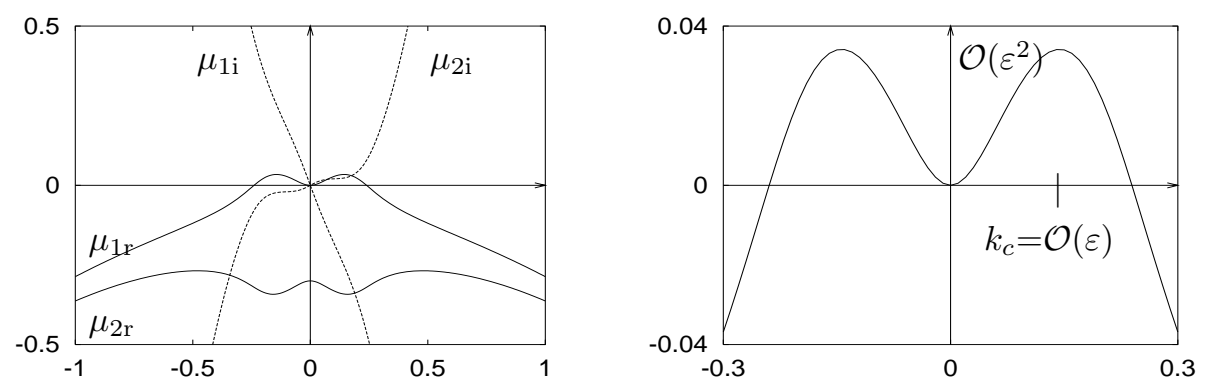

Figure 1: The dispersion relation $\mu_{j}=\mu_{j \mathrm{r}}+\mathrm{i} \mu_{j \mathrm{i}}, j=1,2$, for the IBLe, $\varepsilon=0.2, W=1, \mathrm{R}=10$, $\theta=\pi / 2$; a) the two curves of eigenvalues $\mu_{1,2} ;$ b) blowup of $\mu_{1 \mathrm{r}}(k)$ near $k=0$.

It follows that $A_{0}$ generates an analytic semigroup $e^{t A_{0}}$ with

$$
\left\|e^{t A_{0}} U\right\|_{Y} \leq C \mathrm{e}^{C \varepsilon^{2} t}\|U\|_{Y}
$$

where as phase-space $Y$ we choose, for instance, the Hilbert space $Y=H^{2}(\mathbb{R}) \times H^{1}(\mathbb{R})$ equipped with the norm

$$
\|U\|_{Y}^{2}=\frac{1}{2} \int_{\mathbb{R}}\left\{q^{2}+c_{1} \eta^{2}-2 c_{2} q \eta-2 c_{3} \eta_{x} q+c_{4} q_{x}^{2}+\varepsilon^{-2} \mathrm{~W}\left(\eta_{x}^{2}+c_{4} \eta_{x x}^{2}\right)\right\} \mathrm{d} x .
$$

Here we must choose $c_{2}=2$, and $c_{1}, c_{3}, c_{4}$ can be chosen as

$$
c_{1}=9, \quad c_{3}=-11 \mathrm{R} / 5+2 \cot \theta / 3, \quad c_{4}=\mathrm{R}^{2},
$$

see Section 2.1, where we also motivate the choice of $\|\cdot\|_{Y}$. The strong weighting of derivatives of $\eta$ in (1.9) represents the fact that in (1.6) the small parameter $\varepsilon$ appears in a rather unusual way, namely as an inverse power in front of the damping by the surface tension. This is inherited from the fact that in the underlying Navier-Stokes equations we consider the limit of large surface tension, see Appendix B.2.

Assuming very long waves with a small amplitude the Kuramoto-Sivashinsky equation for the film height $\eta$ can be formally derived from the Navier-Stokes equation. Accordingly, the KSe can also be derived from the IBLe, namely by the ansatz

$$
\eta(t, x)=\varepsilon \eta_{1}(T, X)+\mathcal{O}\left(\varepsilon^{3}\right), \quad q(t, x)=\varepsilon q_{1}(T, X)+\varepsilon^{2} q_{2}(T, X)+\mathcal{O}\left(\varepsilon^{3}\right),
$$


where

$$
T=\varepsilon^{2} t \quad \text { and } \quad X=x-c t
$$

are the very slow time scale and the very long space scale in a frame moving with the speed $c$. These time and space scalings follow directly from the dispersion relation (1.7) for $A_{0}$. Plugging (1.11) into (1.6) we obtain the following hierarchy of equations

$$
\begin{array}{ll}
\mathcal{O}(\varepsilon(1.4)): & q_{1}=2 \eta_{1}, \\
\mathcal{O}\left(\varepsilon^{2}(1.3)\right): & -c \eta_{1 X}=-q_{1 X}=-2 \eta_{1 X} \Rightarrow c=2, \\
\mathcal{O}\left(\varepsilon^{2}(1.4)\right): & -c q_{1 X}=-\frac{8}{5} q_{1 X}+\left(\frac{4}{5}-\frac{2}{\mathrm{R}} \cot \theta\right) \eta_{1 X}+\mathrm{W} \partial_{X}^{3} \eta_{1}-\frac{3}{\mathrm{R}} q_{2}+\frac{6}{\mathrm{R}}\left(\eta_{1} q_{1}-\eta_{1}^{2}\right), \\
& \Rightarrow q_{2}=\left(\frac{8 \mathrm{R}}{15}-\frac{2}{3} \cot \theta\right) \eta_{1 X}+\frac{1}{3} \mathrm{RW} \partial_{X}^{3} \eta_{1}+2 \eta_{1}^{2},
\end{array}
$$

that is, $q_{1}, q_{2}$ are given as functions of $\eta_{1}$. At $\mathcal{O}\left(\varepsilon^{3}(1.3)\right)$ we find $\partial_{T} \eta_{1}=-\partial_{X} q_{2}$ which gives the Kuramoto-Sivashinsky equation

$$
\partial_{T} \eta_{1}=-\left(\frac{8 \mathrm{R}}{15}-\frac{2}{3} \cot \theta\right) \partial_{X}^{2} \eta_{1}-\frac{1}{3} \mathrm{RW} \partial_{X}^{4} \eta_{1}-4 \eta_{1} \partial_{X} \eta_{1}
$$

for $\eta_{1}$. Note that the coefficient of $\partial_{X}^{2} \eta_{1}$ is less than zero iff $\mathrm{R}>\mathrm{R}_{c}$.

Obviously (1.14) is a much simpler equation than (1.6) since it is a semilinear scalar parabolic equation while the IBLe is a quasilinear system. Moreover, the KSe is a generic long wave equation; see, e.g., [11] for a basic review, and, e.g., [12] for the existence and smoothness of solutions $\eta_{1} \in C\left(\left[0, T_{0}\right], H^{m}(\mathbb{R})\right)$ to initial conditions $\eta_{1}(0) \in H^{m}(\mathbb{R})$.

We define the approximation

$$
\varepsilon \psi(t, x)=\left(\begin{array}{c}
\varepsilon q_{1}(T, X)+\varepsilon^{2} q_{2}(T, X) \\
\varepsilon \eta_{1}(T, X)
\end{array}\right)
$$

with $q_{1}, q_{2}$ given by (1.13) and the spaces

$$
H^{r, s}\left(\left(0, t_{0}\right) \times \mathbb{R}\right)=L^{2}\left(\left(0, t_{0}\right), H^{r}(\mathbb{R})\right) \cap H^{s}\left(\left(0, t_{0}\right), L^{2}(\mathbb{R})\right),
$$

and show the following result.

Theorem 1.1 Assume that $\eta_{1} \in C\left(\left[0, T_{0}\right], H^{9}(\mathbb{R})\right)$ is a solution of the KSe. Then for all $C_{1}>0$ there exists $\varepsilon_{0}, C_{2}>0$ such that for all $\varepsilon \in\left(0, \varepsilon_{0}\right)$ the following holds. If

$$
\|U(0, \cdot)-\varepsilon \psi(0, \cdot)\|_{Y} \leq C_{1} \varepsilon^{3 / 2}
$$

then there exist a unique solution $U=(\eta, q)$ of the IBLe,

$$
\eta \in H^{3,3 / 2}\left(\left(0, t_{0}\right) \times \mathbb{R}\right), \quad q \in H^{2,1}\left(\left(0, t_{0}\right) \times \mathbb{R}\right), \quad t_{0}=T_{0} / \varepsilon^{2} .
$$

For $t>0$ the solution is smooth, and it fulfills

$$
\sup _{0 \leq t \leq t_{0}}\|U(t, \cdot)-\varepsilon \psi(t, \cdot)\|_{Y} \leq C_{2} \varepsilon^{3 / 2}
$$


Remark 1.2 The properties of the spaces $H^{r, s}\left(\left(0, t_{0}\right) \times \mathbb{R}\right)$ are reviewed in App.A. Here we remark that $H^{3,3 / 2}\left(\left(0, t_{0}\right) \times \mathbb{R}\right) \times H^{2,1}\left(\left(0, t_{0}\right) \times \mathbb{R}\right) \subset C\left(\left[0, t_{0}\right], Y\right)$ such that (1.18) makes sense. The constants $\varepsilon_{0}, C_{1}, C_{2}$ in Theorem 1.1 depend on $\mathrm{R}$ and $W$ in such a way that for instance $\mathrm{R}_{2} \geq \mathrm{R}_{1}$ gives $\varepsilon_{0}\left(\mathrm{R}_{1}\right) \leq \varepsilon_{0}\left(\mathrm{R}_{2}\right)$ and similar for $C_{1}, C_{2}$, but we do not work this out in detail.

From (1.18) we obtain $\sup _{0 \leq t \leq t_{0}}\|U(t, \cdot)-\varepsilon \psi(t, \cdot)\|_{L^{\infty}} \leq C_{2} \varepsilon^{3 / 2}$. Thus, the error is small compared to the size of the solution. Moreover, the error for $\eta_{x}$ is much smaller, i.e., $\sup _{0 \leq t \leq t_{0}}\left\|\partial_{x} \eta(t, \cdot)-\varepsilon \partial_{x} \psi_{1}(t, \cdot)\right\|_{C^{0}} \leq C_{2} \varepsilon^{7 / 2}$. On the other hand, we must impose the same condition on the initial condition. This means that $\eta_{0}$ must be a long wave in a much stricter sense than $q_{0}$. For $q_{0}$ we may allow small perturbations of $\varepsilon \psi_{1}$ on the original scale. Such 'fast' oscillations in $\eta_{0}$ would violate (1.16). This situation is sketched in figure 2 .

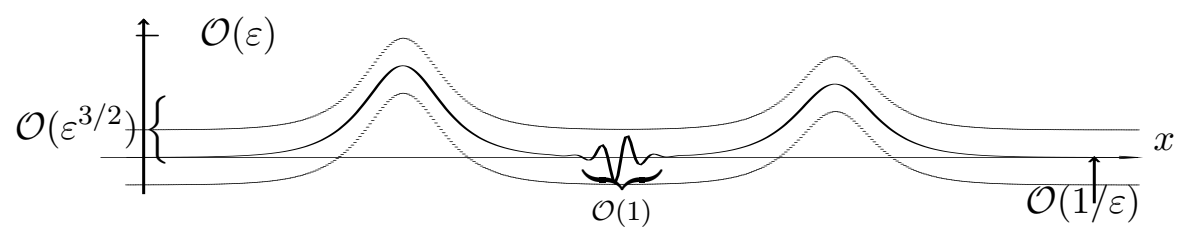

Figure 2: Sketch of initial data. For $q_{0}$ we may allow small oscillations on the original scale.

Remark 1.3 Theorem 1.1 also holds in higher order Sobolev spaces, see Remark 3.2. In fact, if cubic terms were included in (1.6), then in order to control the nonlinearity we would have to work in $H^{3} \times H^{2}$, due to the term $\eta_{x x}^{2} \eta_{x}$, cf. Remark A.5.

Remark 1.4 Our IBLe differs from previously derived hyperbolic Integral Boundary Layer equations $\mathrm{IBL}_{h}$ (see, e.g. [3] and Appendix B.3) in that the linearization of (1.1) around the Nusselt solution is sectorial. Since this is also the case in the free boundary problem for Navier-Stokes equations $[1,21]$, we can use similar methods as will be required to prove an approximation result similar to Theorem 1.1 for the reduction of the Navier-Stokes equation to the KSe.

Remark 1.5 Numerical simulations of the Navier-Stokes equations, of $\mathrm{IBL}_{h}$ and of the KSe suggest that for high but of course finite Weber numbers, corresponding to finite $\varepsilon>0, \mathrm{IBL}_{h}$ is valid as an approximation for the Navier-Stokes equations up to intermediate Reynolds numbers. We conclude that this also holds true for our IBLe (1.6), since (1.6) is derived as a higher order approximation of the Navier-Stokes equations than $\mathrm{IBL}_{h}$. On the other hand the KSe only gives good results for smaller Reynolds numbers; for details see [3] and the references therein. See also [14] for comparison of solutions of the IBLe with experimental results and extensive numerical studies of the inclined film problem.

Remark 1.6 One major reason for the reduction of the inclined film problem to the IBLe or to the KSe is to gain understanding of the formation of solitary and periodic waves on the free surface of the film. For the existence and properties of solitary waves for $\mathrm{IBL}_{h}$ 
and the KS see, again, [3, Section 4] and the references therein. In a somewhat different scaling, a generalized KSe or KdV-KS equation for the free surface can be derived from the Navier-Stokes equations, see [22]. For this KdV-KSe there are analytical results and extensive numerical studies concerning the stability and dynamics of solitary waves, see $[13,6,4]$.

Finally we remark that a result like Theorem 1.1 is not obvious. There are counterexamples where formally derived amplitude equations make wrong predictions about the dynamics in the original system, see $[17,7]$. Moreover, the question which simplified equation, dependent on the parameter regime, still describes the inclined film problem is not settled. Here we contribute to the answer in the sense that for $\mathcal{O}(1)$ Reynolds numbers and in the limit of (very) large Weber number the KSe accurately captures the IBLe dynamics for long waves over the right time scale. We expect the same to be true for the reduction of the NSe to the KSe.

Similarly to our result, the validity of multiple scale approximations to the Navier-Stokes equations in a fixed domain where the instability is located at a finite non-zero wavenumber, has been shown in $[15,18]$. See also, e.g., [19] for the water wave problem, and [16] for such approximation results in simpler settings, i.e., for scalar semilinear parabolic problems.

To explain the difficulty for the proof of Theorem 1.1 we write the IBLe (1.6) as

$$
U_{t}=A_{0} U+B(U, U)
$$

where $B(U, V)$ is a symmetric bilinear form representing the quadratic terms in (1.6). For a $\beta>1$ we set

$$
U=\varepsilon \psi+\varepsilon^{\beta} R
$$

and obtain the equation

$$
\partial_{t} R=A_{0} R+2 \varepsilon B(\psi, R)+\varepsilon^{\beta} B(R, R)+\varepsilon^{-\beta} \operatorname{Res}(\varepsilon \psi)
$$

for the error $R$, where the so called residual

$$
\operatorname{Res}(\varepsilon \psi)=\varepsilon\left(-\partial_{t} \psi+A_{0} \psi+\varepsilon B(\psi, \psi)\right)
$$

contains the terms that do not vanish after inserting (1.11) into (1.6). We essentially have to show a) that solutions to (1.21) for initial conditions $R_{0}=\left.R\right|_{t=0}$ of order $\mathcal{O}(1)$ exist locally, and $\mathrm{b}$ ) that the solutions exist and stay $\mathcal{O}(1)$-bounded up to times $t=T_{0} / \varepsilon^{2}$. In order to show a) for the quasilinear parabolic system (1.21) we use the maximal regularity techniques from [9]. To achieve b) we first define an improved approximation $\varepsilon \tilde{\psi}$ such that the residual is sufficiently small and then derive an energy estimate similar to (1.8). Note that a priori we would expect a growth rate like $C \mathrm{e}^{C \varepsilon t}$ for solutions of $(1.21)$ due to the term $2 \varepsilon B(\tilde{\psi}, R)$ in (1.21). Moreover, because of the term $\varepsilon^{-\beta} \operatorname{Res}(\varepsilon \tilde{\psi})$ we would like to choose $\beta$ small while in order to handle the term $\varepsilon^{\beta} B(R, R)$ we would like to have $\beta$ large. The approach turns out to work with $\beta=3 / 2$. 
In Section 2 we give the calculation leading to the energy estimate (1.8) for the linearized problem and define the improved approximation. The proof of Theorem 1.1 follows in Section 3. The local existence of solutions to (1.6) is shown in Appendix A, which also yields the local existence of solutions to the error equation. In Appendix B we give a brief overview of the physical problem and show how the governing Navier-Stokes equations can be formally reduced to the IBL.

\section{Preparations}

\subsection{The linearized energy estimate}

Here we show the straightforward calculations leading to the energy estimate (1.8). In section 3 we extend these to the quasilinear problem (1.21). We fix $c_{1}=9, c_{2}=2, c_{4}=\mathrm{R}^{2}$ in (1.9) and show how $c_{3}=-11 \mathrm{R} / 5+2 \cot \theta / 3$ yields (1.8). Using

$$
|a b| \leq \delta a^{2}+\frac{1}{4 \delta} b^{2}, \quad \delta>0,
$$

it is clear that, for $\varepsilon$ sufficiently small, $\|\cdot\|_{Y}$ is equivalent to $\|\cdot\|_{Y_{0}}$ with

$$
\|(\eta, q)\|_{Y_{0}}^{2}=\frac{1}{2} \int_{\mathbb{R}} q^{2}+q_{x}^{2}+\eta^{2}+\varepsilon^{-2}\left(\eta_{x}^{2}+\eta_{x x}^{2}\right) \mathrm{d} x
$$

and hence a norm on $H^{2}(\mathbb{R}) \times H^{1}(\mathbb{R})$. By Fourier transform it is obvious that the solution $U$ of the linearized equation $U_{t}=A_{0} U$ with $A_{0}$ from (1.6) exists and is smooth. We then obtain

$$
\begin{aligned}
& \frac{\mathrm{d}}{\mathrm{d} t}\|U\|_{Y}^{2}=\int_{\mathbb{R}}\left\{( q - 2 \eta - c _ { 3 } \eta _ { x } - \mathrm { R } ^ { 2 } q _ { x x } ) \left[\frac{6}{\mathrm{R}} \eta+\left(\frac{4}{5}-\frac{2}{\mathrm{R}} \cot \theta\right) \eta_{x}-\frac{3}{\mathrm{R}} \eta_{x x}\right.\right. \\
& \left.+\varepsilon^{-2} \mathrm{~W} \eta_{x x x}-\frac{3}{\mathrm{R}} q-\frac{8}{5} q_{x}+\frac{7}{2 \mathrm{R}} q_{x x}\right] \\
& \left.-9 \eta q_{x}+\varepsilon^{-2} \mathrm{~W}\left(\eta_{x x} q_{x}+\mathrm{R}^{2} \eta_{x x x} q_{x x}\right)-c_{3} q_{x}^{2}\right\} \mathrm{d} x \\
& =\int_{\mathbb{R}}\left\{-\frac{3}{\mathrm{R}} q^{2}+\frac{12}{\mathrm{R}} q \eta-\frac{12}{\mathrm{R}} \eta^{2}+\left(\frac{4}{5}-\frac{2}{\mathrm{R}} \cot \theta-\frac{16}{5}+9+\frac{3}{\mathrm{R}} c_{3}\right) \eta_{x} q\right. \\
& +\left(-c_{3}-3 \mathrm{R}-\frac{7}{2 \mathrm{R}}\right) q_{x}^{2}+\left(6 \mathrm{R}+\frac{8}{5} c_{3}+\frac{10}{\mathrm{R}}\right) q_{x} \eta_{x}+\left(-c_{3}\left(\frac{4}{5}-\frac{2}{3} \cot \theta\right)-\frac{6}{\mathrm{R}}\right) \eta_{x}^{2} \\
& \left.+\left(\mathrm{R}^{2}\left(\frac{4}{5}-\frac{2}{3} \cot \theta\right)+\frac{7 c_{3}}{2 \mathrm{R}}\right) q_{x} \eta_{x x}-\frac{7 \mathrm{R}}{2} q_{x x}^{2}+3 \mathrm{R} q_{x x} \eta_{x x}+\varepsilon^{-2} \mathrm{~W} c_{3} \eta_{x x}^{2}\right\} \mathrm{d} x .
\end{aligned}
$$

The quadratic form in $\eta, q$ without derivatives is nonpositive. For $c_{3}=-11 \mathrm{R} / 5+2 \cot \theta / 3$ the coefficient of $\eta_{x} q$ vanishes. Moreover, the coefficients of $q_{x}^{2}, q_{x x}^{2}$ are negative definite and the coefficient of $\eta_{x x}^{2}$ is negative definite with strong weight $\varepsilon^{-2}$. Note that $c_{3}<0$ due to (1.2). The terms with $\eta_{x}, q_{x}$ yield $\frac{\mathrm{d}}{\mathrm{d} t}\|U\|_{Y}^{2} \leq C_{1}\left\|\eta_{x}\right\|_{L^{2}}^{2}$, but since $\eta_{x}^{2}$ appears in $\|U\|_{Y}$ with weight $\varepsilon^{-2}$, we nevertheless obtain

$$
\frac{\mathrm{d}}{\mathrm{d} t}\|U\|_{Y}^{2} \leq C_{1} \varepsilon^{2}\|U\|_{Y}^{2}-C\left\|q_{x}\right\|_{H^{1}}^{2} .
$$


On the other hand the coefficient of $q \eta_{x}$ has to vanish identically since we have no negative definite term in $q^{2}$, and can not have one as is clear from the dispersion relation. Therefore we have to introduce $c_{3}$ in (1.9). From (2.2) we get (1.8) using Gronwall's lemma. The dissipation in $q$ in (2.2) will be important for the quasilinear problem (1.21).

\subsection{The residual}

For notational convenience and without loss of generality for our purposes we assume in the following that we have a vertically falling film such that $\cot \theta=0$. Then the critical Reynolds number is $R_{c}=0$, and we may further assume w.l.o.g. that

$$
\mathrm{R}=W=1 .
$$

In order to get a small residual in (1.21) we define an improved approximation by

$$
\varepsilon \tilde{\psi}(t, x)=\left(\begin{array}{c}
\varepsilon \eta_{1}(T, X) \\
\sum_{j=1}^{3} \varepsilon^{j} q_{j}(T, X)
\end{array}\right), \quad T=\varepsilon^{2}, \quad X=\varepsilon(x-2 t) .
$$

Plugging (2.4) into (1.3),(1.4) we first obtain (1.13) and (1.14) as before, and then

$$
\begin{array}{r}
\mathcal{O}\left(\varepsilon^{3}(1.4)\right): \quad q_{1 T}-2 q_{2 X}=\frac{8}{5} \eta_{1 X} \eta_{1}+\frac{7}{2} q_{1 X X}+\eta_{1} \eta_{1 X X X}-3 \eta_{1 X X}-3 q_{3} \\
+6 \eta_{1} q_{2}-\frac{8}{5} q_{2 X}-\frac{12}{5} q_{1} q_{1 X}+\frac{8}{5} \eta_{1} \eta_{1 X} \\
\Rightarrow q_{3}=\frac{1}{3}\left(\frac{4}{5} \partial_{X}^{4} \eta_{1}+3 \eta_{1 X} \eta_{1}+\frac{52}{25} \eta_{1 X X} \eta_{1}+\frac{112}{5} \eta_{1 X} \eta_{1}+12 \eta_{1}^{3}\right) .
\end{array}
$$

With $q_{3}$ given by (2.5) all terms up to order $\mathcal{O}\left(\varepsilon^{3}\right)$ vanish in the residual

$$
\operatorname{Res}(\varepsilon \tilde{\psi})=\varepsilon^{4} f=\varepsilon^{4}\left(\begin{array}{l}
f_{1} \\
f_{2}
\end{array}\right) .
$$

To leading order in derivatives we have

$$
\begin{aligned}
& f_{1}=-q_{3 X}=-\frac{4}{15} \partial_{X}^{5} \eta_{1}+\tilde{f}_{1} \\
& f_{2}=-\varepsilon q_{3 T}+\tilde{f}_{2}=-\frac{4 \varepsilon}{15} \partial_{X}^{4} \partial_{T} \eta_{1}+\tilde{f}_{2}=\frac{4 \varepsilon}{45} \partial_{X}^{8} \eta_{1}+\tilde{f}_{2} .
\end{aligned}
$$

Later we need

$$
\left(f_{1}, f_{2}\right) \in C\left(\left[0, T_{0} / \varepsilon^{2}\right], H^{2}(\mathbb{R}) \times H^{1}(\mathbb{R})\right)
$$

and therefore

$$
\eta_{1} \in C\left(\left[0, T_{0}\right], H^{9}(\mathbb{R})\right)
$$

in Theorem 1.1. The (nonlinear) functions $\tilde{f}_{1,2}$ in (2.7) contain lower order derivatives of $\eta_{1}$ and it can be easily checked that (2.8) holds if (2.9) does. In order to estimate the residual in $Y$ 
we finally need to take care of how scaling affects the $L^{2}$ norm, i.e., $\|u(\varepsilon \cdot)\|_{L^{2}}=\varepsilon^{-1 / 2}\|u(\cdot)\|_{L^{2}}$. This loss of $\varepsilon^{-1 / 2}$ is the reason why we can not choose $\beta=2$ in (1.20), which would be more convenient in order to control the nonlinear terms in (1.21). We summarize our results as follows.

Lemma 2.1 Assume that $\eta_{1} \in C\left(\left[0, T_{0}\right], H^{9}(\mathbb{R})\right)$. Then

$$
\sup _{0 \leq t<T_{0} / \varepsilon^{2}}\|\varepsilon \tilde{\psi}-\varepsilon \psi\|_{Y} \leq C \varepsilon^{5 / 2} \quad \text { and } \sup _{0 \leq t \leq T_{0} / \varepsilon^{2}}\|\operatorname{Res}(\varepsilon \tilde{\psi})\|_{Y} \leq C \varepsilon^{7 / 2}
$$

Due to the first estimate in Lemma 2.1 we can use $\varepsilon \tilde{\psi}$ instead of $\varepsilon \psi$ in the proof of Theorem 1.1, and in order not to proliferate symbols we drop the ${ }^{\sim}$ in the following. Also we write $\psi=\left(\psi_{1}, \psi_{2}\right)$ and $\psi_{j}^{\prime}=\partial_{X} \psi_{j}$.

\section{Proof of Theorem 1.1}

From the local existence of solutions to the IBLe in Theorem A.1 we directly obtain the following local existence of solutions to (1.21).

Corollary 3.1 Let $R_{0} \in H^{2}(\mathbb{R}) \times H^{1}(\mathbb{R})$ and $0<t_{1} \leq T_{0} / \varepsilon^{2}$. Then there exists an $\varepsilon_{1}>0$ such that for all $\varepsilon \in\left(0, \varepsilon_{1}\right)$ there exists a unique solution $R \in H^{3,3 / 2}\left(\left(0, t_{1}\right) \times \mathbb{R}\right) \times H^{2,1}\left(\left(0, t_{1}\right) \times\right.$ $\mathbb{R})$ of the error equation (1.21) with $R(0)=R_{0}$.

Proof. For $\varepsilon_{1}$ sufficiently small we have

$$
\left\|U_{0}\right\|_{H^{2} \times H^{1}}=\left\|\left.\left(\varepsilon \psi+\varepsilon^{3 / 2} R\right)\right|_{t=0}\right\|_{H^{2} \times H^{1}} \leq \rho
$$

for all $\varepsilon \in\left(0, \varepsilon_{1}\right)$, with $\rho>0$ from Theorem A.1. Therefore there exists a unique solution $U \in H^{3,3 / 2}\left(\left(0, t_{1}\right) \times \mathbb{R}\right) \times H^{2,1}\left(\left(0, t_{1}\right) \times \mathbb{R}\right)$ of $(1.6)$. Using the smoothness of $\eta_{1}$ we find that the solution $R=\varepsilon^{-3 / 2}(U-\varepsilon \psi)$ of (1.21) has the same regularity.

The proof of Theorem 1.1 now works as follows: due to Corollary 3.1 we have a local solution $R \in C\left(\left[0, t_{1}\right), H^{2} \times H^{1}\right)$ of (1.21). Thus we may choose $t_{1}$ so small that $\sup _{0 \leq t \leq t_{1}}\|R\|_{Y} \leq 2\left\|R_{0}\right\|_{Y} \leq 2 C_{1}$. This implies

$$
\sup _{0 \leq t \leq t_{1}}\left(\|r\|_{\infty}+\|\xi\|_{\infty}+\varepsilon^{-1}\left\|\partial_{x} \xi\right\|_{\infty}\right) \leq 2 C C_{1}
$$

Using this, we derive an energy estimate that implies $\left\|R\left(t_{1}\right)\right\|_{Y} \leq C \mathrm{e}^{C \varepsilon^{2} t_{1}}\left\|R_{0}\right\|_{Y}$. Thus, using Corollary 3.1 again, the solution can be continued and stays $\mathcal{O}(1)$ bounded in $Y$ until $t_{1}=$ $t_{0}=T_{0} / \varepsilon^{2}$.

It will be convenient to write (1.21) as

$$
R_{t}=A(t, R) R+\varepsilon^{2} f
$$


where, with $a_{0}, \ldots, b_{1}$ from (1.5),

$$
\begin{aligned}
& R=\left(\begin{array}{c}
\xi \\
r
\end{array}\right), \quad A(t, R)=\left(\begin{array}{cc}
0 & -\partial_{x} \\
\tilde{a}_{0}+\tilde{a}_{1} \partial_{x}+\tilde{a}_{2} \partial_{x}^{2}+\varepsilon^{-2} \tilde{a}_{3} \partial_{x}^{3} & -\tilde{b}_{0}-\tilde{b}_{1} \partial_{x}+\tilde{b}_{2} \partial_{x}^{2}
\end{array}\right), \\
& \tilde{a}_{0}=\tilde{a}_{0}(t, \xi)=a_{0}\left(\varepsilon \psi_{1}+\varepsilon^{3 / 2} \xi\right)-\frac{8}{5} \varepsilon^{2} \psi_{1}^{\prime}+\frac{9}{2} \varepsilon^{3} \psi_{1}^{\prime \prime}+\varepsilon^{4} \psi_{1}^{\prime \prime \prime}+6 \varepsilon \psi_{2}+\frac{8}{5} \varepsilon^{2} \psi_{2}^{\prime}+3 \varepsilon^{3} \psi_{2}^{\prime \prime \prime} \\
& \tilde{a}_{1}=\tilde{a}_{1}(t, R)=a_{1}\left(\varepsilon \psi+\varepsilon^{3 / 2} R\right)-2 \varepsilon^{2} \psi_{1}^{\prime}, \quad \tilde{a}_{2}=\tilde{a}_{2}(t, \xi)=a_{2}\left(\varepsilon \psi_{1}+\varepsilon^{3 / 2} \xi\right), \\
& \tilde{a}_{3}=\tilde{a}_{3}(t, \xi)=a_{3}\left(\varepsilon \psi_{1}+\varepsilon^{3 / 2} \xi\right), \\
& \tilde{b}_{0}=\tilde{b}_{0}(t, \xi)=b_{0}\left(\varepsilon \psi_{1}+\varepsilon^{3 / 2} \xi\right)-\frac{8}{5} \varepsilon^{2} \psi_{1}^{\prime}+\frac{12}{5} \varepsilon^{2} \psi_{2}^{\prime}+\frac{9}{2} \varepsilon^{3} \psi_{1}^{\prime \prime}, \\
& \tilde{b}_{1}=\tilde{b}_{1}(t, R)=b_{1}\left(\varepsilon \psi+\varepsilon^{3 / 2} R\right)-\varepsilon^{2} \psi_{1}^{\prime}, \quad \tilde{b}_{2}=b_{2}=7 / 2 .
\end{aligned}
$$

The main idea to obtain the energy estimate is to define an equivalent norm $N_{Y}(R, t)$ on $Y$ that depends on time and the solution itself in such a way, that the high order and strongly weighted mixed product as $\varepsilon^{-2} \partial_{x}^{2} q \partial_{x}^{3} \eta$ still cancel after integration by parts in $\frac{\mathrm{d}}{\mathrm{d} t}\|R\|_{N_{Y}(R, t)}^{2}$. This can be achieved by dividing all terms in (1.9) involving $r$ by $\tilde{a}_{3}$. Moreover, we need correction terms that eliminate terms of order $\mathcal{O}(\varepsilon)$ and $\mathcal{O}\left(\varepsilon^{3 / 2}\right)$ in $\frac{\mathrm{d}}{\mathrm{d} t}\|R\|_{N(t, R)}$ without derivatives that come from $2 \varepsilon B(\tilde{\psi}, R)+\varepsilon^{3 / 2} B(R, R)$ in (1.21).

Thus, with coefficients $\gamma_{1}, \ldots, \gamma_{4} \in \mathbb{R}$ to be determined, we define

$$
\begin{gathered}
\|R\|_{N_{Y}(t, R)}^{2}=E+F_{1}+F_{2}, \\
E=\frac{1}{2} \int_{\mathbb{R}}\left\{\frac{1}{\tilde{a}_{3}}\left[r^{2}-4 r \xi-2 c_{3} r \xi_{x}+r_{x}^{2}\right]+9 \xi^{2}+\varepsilon^{-2}\left[\xi_{x}^{2}+\xi_{x x}^{2}\right]\right\} \mathrm{d} x, \\
F_{1}=\int \frac{1}{\tilde{a}_{3}} \varepsilon \eta_{1}\left[\gamma_{1} r^{2}+\gamma_{2} \xi r\right] \mathrm{d} x, \quad F_{2}=\int \frac{1}{\tilde{a}_{3}} \varepsilon^{3 / 2} r\left[\gamma_{3} r \xi+\gamma_{4} \xi^{2}\right] \mathrm{d} x,
\end{gathered}
$$

where for notational convenience we keep writing $c_{3}$ for $-11 / 5$. Due to (3.1) we have

$$
1-C \varepsilon \leq \sup _{0 \leq t \leq t_{0}, x \in \mathbb{R}}\left|a_{3}\right|=\sup _{0 \leq t \leq t_{0}, x \in \mathbb{R}}\left|1+\varepsilon \psi_{1}+\varepsilon^{3 / 2} \xi\right| \leq 1+C \varepsilon
$$

Therefore $N_{Y}(t, R)$ is still an equivalent norm on $Y$ if $\varepsilon$ is sufficiently small. Moreover, space and time derivatives of $\tilde{a}_{0}, \tilde{a}_{1}, \ldots, \tilde{b}_{2}$ produce terms of order $\mathcal{O}\left(\varepsilon^{3 / 2}\right)$, and in particular we have

$$
\begin{aligned}
& \frac{\mathrm{d}}{\mathrm{d} x} \tilde{a}_{0}=6 \varepsilon^{3 / 2} \xi_{x}+\varepsilon^{2} h_{1}, \quad \frac{\mathrm{d}}{\mathrm{d} x} \tilde{b}_{0}=3 \varepsilon^{3 / 2} \xi_{x}+\varepsilon^{2} h_{2}, \\
& \frac{\mathrm{d}}{\mathrm{d} x} \tilde{a}_{3}=\varepsilon^{3 / 2} \xi_{x}+\varepsilon^{2} h_{3}, \quad \frac{\mathrm{d}}{\mathrm{d} t} \tilde{a}_{3}=\varepsilon^{3 / 2} \xi_{t}+\varepsilon \frac{\mathrm{d}}{\mathrm{d} t} \psi_{1}=-\varepsilon^{3 / 2} r_{x}+h_{4},
\end{aligned}
$$

with $\left\|h_{j}\right\|_{L^{\infty}}=\mathcal{O}\left(\varepsilon^{2}\right), j=1,2,3,4$. Hence we can estimate terms like for instance $\left(\frac{\mathrm{d}}{\mathrm{d} x} \tilde{\tilde{b}}_{0}\right) r \xi$ that show up during integration by parts in $\frac{\mathrm{d}}{\mathrm{d} t}\|R\|_{N(t, R)}^{2}$ as

$$
\begin{aligned}
\int \frac{\mathrm{d}}{\mathrm{d} x}\left(\frac{\tilde{b}_{0}}{\tilde{a}_{3}}\right) r \xi \mathrm{d} x & =\int \frac{1}{3 \tilde{a}_{3}}\left(\varepsilon^{3 / 2} \xi_{x} r \xi\right)-6 \frac{\tilde{b}_{0}}{\tilde{a}_{3}^{2}}\left(\varepsilon^{3 / 2} r_{x} r \xi\right)+\mathcal{O}\left(\varepsilon^{2}\right)|r \xi| \mathrm{d} x \\
& \leq C\|\xi\|_{\infty} \int \varepsilon \xi_{x}^{2}+\varepsilon r_{x}^{2}+\varepsilon^{2}\left(\xi^{2}+r^{2}\right) \mathrm{d} x+C \varepsilon^{2} \int r^{2}+\xi^{2} \mathrm{~d} x
\end{aligned}
$$


and similar for $\left(\frac{\mathrm{d}}{\mathrm{d} t} \frac{1}{\tilde{a}_{3}}\right)\left(r^{2}-4 r \xi\right)$, see (3.10). The first term on the right hand side of (3.7) is estimated by $\varepsilon^{3} \varepsilon^{-2} \xi_{x}^{2}$ and the second term is well behaved since we will have an $\mathcal{O}(1)$ negative definite term $-C r_{x}^{2}$ in $\frac{\mathrm{d}}{\mathrm{d} t}\|R\|_{N_{Y}(t, R)}^{2}$. This is essentially the first reason why the estimate (2.2) can be carried over to the quasilinear problem (3.2). The second reason is, that the coefficients $\gamma_{1}, \ldots, \gamma_{4}$ can be chosen in such a way that the terms $r^{2}, \xi^{2}$ without derivatives in $\frac{\mathrm{d}}{\mathrm{d} t}\|R\|_{N_{Y}(t, R)}^{2}$ have $\mathcal{O}\left(\varepsilon^{2}\right)$ coefficients. This is possible again due to the fact that the small parameter $\varepsilon$ does not as usual enter (1.6) as a coefficient of the low order terms but in inverse power as coefficient of the high order damping term.

We start with $\frac{\mathrm{d}}{\mathrm{d} t} E$. Using $2 \int g f_{x} f \mathrm{~d} x=-\int g_{x} f^{2} \mathrm{~d} x$ we obtain

$$
\begin{gathered}
\frac{\mathrm{d}}{\mathrm{d} t} E=d_{1}+d_{2}+d_{3}+d_{4}, \\
d_{1}=\int\left\{\left[r-2 \xi-c_{3} \xi_{x x}-r_{x x}-\tilde{a}_{3} \frac{\mathrm{d}}{\mathrm{d} x}\left(1 / \tilde{a}_{3}\right) r_{x}\right]\right. \\
\left.\left[\frac{\tilde{a}_{0}}{\tilde{a}_{3}} \xi+\frac{\tilde{a}_{1}}{\tilde{a}_{3}} \xi_{x}+\frac{\tilde{a}_{2}}{\tilde{a}_{3}} \xi_{x x}+\varepsilon^{-2} \xi_{x x x}-\frac{\tilde{b}_{0}}{\tilde{a}_{3}} r-\frac{\tilde{b}_{1}}{\tilde{a}_{3}} r_{x}+\frac{\tilde{b}_{2}}{\tilde{a}_{3}} r_{x x}+\varepsilon^{2} \frac{f_{2}}{\tilde{a}_{3}}\right]\right\} \mathrm{d} x, \\
d_{2}=\int-9 \xi r_{x}+\varepsilon^{-2}\left(\xi_{x x} r_{x}+\xi_{x x x} r_{x x}\right)+9 \xi \varepsilon^{2} f_{1}+\varepsilon^{-2} \varepsilon^{2}\left(\xi_{x} f_{1 x}+\xi_{x x} f_{1 x x}\right) \mathrm{d} x, \\
d_{3}=\int \frac{1}{\tilde{a}_{3}}\left[2 r r_{x}+c_{3} r r_{x x}\right] \mathrm{d} x, \quad d_{4}=\int\left(\frac{\mathrm{d}}{\mathrm{d} t} \frac{1}{\tilde{a}_{3}}\right)\left[r^{2}-4 r \xi-2 c_{3} r \xi_{x}+r_{x}^{2}\right] \mathrm{d} x .
\end{gathered}
$$

Integrations by parts yields

$$
\begin{aligned}
d_{1}+d_{2}+d_{3} & =\int\left\{\frac{1}{\tilde{a}_{3}}\left[-\tilde{b}_{0} r^{2}+\left(\tilde{a}_{0}+2 \tilde{b}_{0}\right) r \xi-2 \tilde{a}_{0} \xi^{2}\right]+\mathcal{O}\left(\varepsilon^{2}\right)\left(r^{2}+\xi^{2}\right)\right. \\
& +\frac{1}{\tilde{a}_{3}}\left[\tilde{a}_{1}-2 \tilde{b}_{1}+9+c_{3} \tilde{b}_{0}+\mathcal{O}(\varepsilon)\right] r \xi_{x}+\frac{1}{\tilde{a}_{3}}\left[-c_{3}-\tilde{b}_{0}-\tilde{b}_{2}+\mathcal{O}(\varepsilon)\right] r_{x}^{2} \\
& +\frac{1}{\tilde{a}_{3}}\left[\tilde{a}_{0}+c_{3} \tilde{b}_{1}-\tilde{a}_{2}+2 \tilde{b}_{2}+\mathcal{O}(\varepsilon)\right] r_{x} \xi_{x}+\frac{1}{\tilde{a}_{3}}\left[-c_{3} \tilde{a}_{1}+2 \tilde{a}_{2}+\mathcal{O}(\varepsilon)\right] \xi_{x}^{2} \\
& +\left[c_{3} \varepsilon^{-2}+\mathcal{O}(\varepsilon)\right] \xi_{x x}^{2}+\frac{1}{\tilde{a}_{3}}\left[\tilde{a}_{1}+c_{3} \tilde{b}_{2}+\mathcal{O}(\varepsilon)\right] r_{x} \xi_{x x}-\frac{\tilde{b}_{2}}{\tilde{a}_{3}} r_{x x}^{2}-\frac{2 \tilde{a}_{2}}{\tilde{a}_{3}} r_{x x} \xi_{x x} \\
& \left.+\varepsilon^{2} f_{2}\left[r-2 \xi-c_{3} \xi_{x x}-r_{x x}+\mathcal{O}(\varepsilon) r_{x}\right]+9 \varepsilon^{2} f_{1} \xi+f_{1 x} \xi_{x}+f_{1 x x} \xi_{x x}\right\} \mathrm{d} x
\end{aligned}
$$

where the order symbol $\mathcal{O}(\varepsilon)$ always refers to terms estimated in $L^{\infty}$. The coefficient of $r \xi_{x}$ in (3.9) is $\mathcal{O}(\varepsilon)$ due to the choice of $c_{3}$ and (3.1). Similar to (3.7), $d_{4}$ can be estimated as

$$
\begin{aligned}
d_{4} \leq & C\|r\|_{\infty} \int \varepsilon^{3 / 2}\left(\left|r_{x} r\right|+\left|r_{x} \xi\right|\right) \mathrm{d} x+C \varepsilon^{2} \int r^{2}+\xi^{2} \mathrm{~d} x \\
& +C \varepsilon^{3 / 2}\left\|r_{x}\right\|_{\infty} \int\left[-2 c_{3} r \xi_{x}+r_{x}^{2}\right] \mathrm{d} x .
\end{aligned}
$$

and therefore (3.9) and (3.10), except of the first term on the right hand side of (3.9), can be estimated by $C \varepsilon^{2} E+C_{\text {res }} \varepsilon^{2}$. 
Thus, we now have

$$
\begin{aligned}
\frac{\mathrm{d}}{\mathrm{d} t}\|R\|_{N_{Y}(t, R)}^{2} & =\frac{\mathrm{d}}{\mathrm{d} t} E+\frac{\mathrm{d}}{\mathrm{d} t} F_{1}+\frac{\mathrm{d}}{\mathrm{d} t} F_{2} \\
& \leq \int \frac{1}{\tilde{a}_{3}}\left[-\tilde{b}_{0} r^{2}+\left(\tilde{a}_{0}+2 \tilde{b}_{0}\right) r \xi-2 \tilde{a}_{0} \xi^{2}\right] \mathrm{d} x+\frac{\mathrm{d}}{\mathrm{d} t} F_{1}+\frac{\mathrm{d}}{\mathrm{d} t} F_{2}+C \varepsilon^{2} E+\varepsilon^{2} C_{\text {res }} .
\end{aligned}
$$

To control the first three terms on the right hand side of (3.11) we calculate

$$
\begin{aligned}
\frac{\mathrm{d}}{\mathrm{d} t} F_{1} & =\int \frac{\varepsilon \eta_{1}}{\tilde{a}_{3}}\left[2 \gamma_{1} r(6 \xi-3 r)+\gamma_{2} \xi(6 \xi-3 r)\right] \mathrm{d} x+h_{1}, \\
\frac{\mathrm{d}}{\mathrm{d} t} F_{2} & =\int \frac{\varepsilon^{3 / 2} \xi}{\tilde{a}_{3}}\left[2 \gamma_{2} r(6 \xi-3 r)+\gamma_{4} \xi(6 \xi-3 r)\right] \mathrm{d} x+h_{2},
\end{aligned}
$$

where $h_{1}$ and $h_{2}$ contain terms like for instance $h_{1}=-\varepsilon \eta_{1} r_{x} r^{2} / \tilde{a}_{3}+\ldots$ that can be controlled by the negative definite terms in $\frac{\mathrm{d}}{\mathrm{d} t} E$ as in (3.7) and (3.10). Since

$$
\tilde{b}_{0}=3-6 \varepsilon \eta_{1}-6 \varepsilon^{3 / 2} \xi+\mathcal{O}\left(\varepsilon^{2}\right), \quad \tilde{a}_{0}=6-18 \varepsilon \eta_{1}-6 \varepsilon^{3 / 2} \xi+\mathcal{O}\left(\varepsilon^{2}\right),
$$

we thus obtain

$$
\begin{aligned}
& \frac{\mathrm{d}}{\mathrm{d} t}\|R\|_{N_{Y}(t, R)}^{2} \leq \int \frac{1}{\tilde{a}_{3}}\left\{\left(-3+6 \varepsilon\left(1-\gamma_{1}\right) \eta_{1}+6 \varepsilon^{3 / 2} \xi\left(1-\gamma_{3}\right) \varepsilon^{3 / 2} \xi\right) r^{2}\right. \\
&+\left(12-3\left(10-4 \gamma_{1}+\gamma_{2}\right) \varepsilon \eta_{1}-3\left(6-4 \gamma_{3}+\gamma_{4}\right) \varepsilon^{3 / 2} \xi\right) r \xi \\
&\left.-\left(12-6\left(6+\gamma_{2}\right) \varepsilon \eta_{1}-6\left(2+\gamma_{4}\right) \varepsilon^{3 / 2} \xi\right) \xi^{2}\right\} \mathrm{d} x \\
&+C \varepsilon^{2} E+C_{\mathrm{res}} \varepsilon^{2} .
\end{aligned}
$$

Chosing $\gamma_{1}=1, \gamma_{2}=-6, \gamma_{3}=1, \gamma_{4}=-2$ the $\mathcal{O}(\varepsilon)$ and $\mathcal{O}\left(\varepsilon^{3 / 2}\right)$ coefficients in the integral vanish, and since $\int \frac{1}{\tilde{a}_{3}}\left[-3 r^{2}+12 r \xi-12 \xi^{2}\right] \mathrm{d} x \leq 0$ we finally obtain

$$
\frac{\mathrm{d}}{\mathrm{d} t}\|R\|_{N_{Y}(t, R)}^{2} \leq C \varepsilon^{2} E+C_{\mathrm{res}} \varepsilon^{2} \leq C \varepsilon^{2}\|R\|_{N_{Y}(t, R)}^{2}+C_{\mathrm{res}} \varepsilon^{2}
$$

This gives $\|R\|_{N_{Y}(t, R)}^{2} \leq C \mathrm{e}^{C \varepsilon^{2} t}\left\|\left.R\right|_{t=0}\right\|_{N_{Y}(t, R)}^{2}+C_{\text {res }} \mathrm{e}^{C \varepsilon^{2} t}$ using Gronwall's lemma. Setting $C_{2}=C \mathrm{e}^{C T_{0} / 2} C_{1}+C_{\text {res }} \mathrm{e}^{C T_{0} / 2}$, the proof of Theorem 1.1 is complete.

Remark 3.2 Theorem 1.1 also holds in higher order Sobolev spaces. For $m \geq 3$ we can define $Y_{m}=H^{m}(\mathbb{R}) \times H^{m-1}(\mathbb{R})$ with $\|\cdot\|_{Y_{m}}$ defined in a similar way as $\|\cdot\|_{Y}$, i.e., for $\cot \theta=0, \mathrm{R}=\mathrm{W}=1$,

$$
\|U\|_{Y_{3}}^{2}=\frac{1}{2} \int_{\mathbb{R}}\left\{\begin{array}{l}
q^{2}+9 \eta^{2}-4 q \eta-\frac{22}{5} \eta_{x} q+2 q_{x} \eta_{x x}+q_{x}^{2} \\
\\
+q_{x x}^{2}+2 q_{x} \eta_{x x}+\varepsilon^{-2}\left(\eta_{x}^{2}+\eta_{x x}^{2}+\eta_{x x x}^{2}\right)
\end{array}\right\} \mathrm{d} x .
$$

Then for $\|U(0, \cdot)-\varepsilon \psi(0, \cdot)\|_{Y_{m}} \leq C_{1} \varepsilon^{3 / 2}$ and $\eta_{1} \in H^{m+6}(\mathbb{R})$ we obtain a solution $U \in$ $H^{m+1,(m+1) / 2} \times H^{m, m / 2}$ with $\sup _{0 \leq t \leq t_{0}}\|U(t, \cdot)-\varepsilon \psi(t, \cdot)\|_{Y_{m}} \leq C_{2} \varepsilon^{3 / 2}$. The local existence of solutions in these higher order spaces is already shown in Theorem A.1, and from the above proof it can be seen that the high order terms are uncritical in the energy estimates. 


\section{A Local existence of solutions for the IBLe}

To treat the initial value problem for the IBLe (1.6) we use the spaces

$$
H^{r, s}=H^{r, s}\left(\left(0, t_{0}\right) \times \mathbb{R}\right)=L^{2}\left(\left(0, t_{0}\right), H^{r}(\mathbb{R})\right) \cap H^{s}\left(\left(0, t_{0}\right), L^{2}(\mathbb{R})\right),
$$

defined for $r, s \geq 0$. Because we have a parabolic system we will always have $s=r / 2$ and therefore we introduce the notation $K^{r}=K^{r}\left(\left(0, t_{0}\right) \times \mathbb{R}\right)=H^{r, r / 2}\left(\left(0, t_{0}\right) \times \mathbb{R}\right)$. We recall a few facts on the spaces $H^{r, r / 2}\left(\left(0, t_{0}\right) \times \mathbb{R}\right)$, mainly from [9].

If $u \in H^{r, s}$ and $j, k \in \mathbb{N}$ with $1-(j / r+k / s) \geq 0$, then $\partial_{t}^{k} \partial_{x}^{j} u \in H^{\mu, \nu}$ with $\mu / r=\nu / s=1-(j / r+k / s)$, [9, Prop.4.2.3]. Especially, if $u \in K^{r}$ and $1-(j / r+2 k / r) \geq 0$, then $\partial_{t}^{k} \partial_{x}^{j} u \in K^{r-j-2 k}$. For $k<r / 2-1 / 2$ we have traces $\partial_{t}^{k} u(0, \cdot) \in H^{r-2 k-1}(\mathbb{R})$, [9, Prop.4.2.1]. Conversely, if these traces are given at $t=0$, then there exists a bounded extension operator such that $u \in K^{r},[9$, Thm.4.2.3]. Similarly, there exists a bounded extension operator from $K^{r}=K^{r}\left(\left(0, t_{0}\right) \times \mathbb{R}\right)$ into $K^{r}(\mathbb{R} \times \mathbb{R}),[21$, Lemma 3.1].

For $u \in K^{r}\left(\mathbb{R} \times \mathbb{R}^{n}\right)$ let $\hat{\hat{u}}(\tau, k)=\iint \mathrm{e}^{-\mathrm{i}(\tau t+k \cdot x)} u(t, x) \mathrm{d} k \mathrm{~d} t$ be the Fourier transform in time and space of $u$. Then we have the equivalence of norms

$$
\|u\|_{K^{r}\left(\mathbb{R} \times \mathbb{R}^{n}\right)}^{2} \sim \iint|\hat{\hat{u}}(\tau, \xi)|^{2}\left(1+|k|^{2}+|\tau|\right)^{r} \mathrm{~d} k \mathrm{~d} \tau .
$$

From this follows easily that if $u \in K^{r}\left(\mathbb{R} \times \mathbb{R}^{n}\right)$ with $r>(n+2) / 2$, then $u$ is bounded and continuous. Finally we need the special subspace

$$
K_{0}^{r}=K_{0}^{r}\left(\left(0, t_{0}\right) \times \mathbb{R}\right)=\left\{u \in K^{r}\left(\left(0, t_{0}\right) \times \mathbb{R}\right): \partial_{t}^{k} u(0, \cdot)=0 \text { for } k \in \mathbb{N}, k<r / 2-1 / 2\right\} .
$$

For $u \in K_{0}^{r}((0, \infty) \times \mathbb{R})$ the continuation by $u(t)=0$ for $t<0$ is in $K^{r}(\mathbb{R} \times \mathbb{R}),[9$, Thm. 1.11.5]. Additional to the full space-time transform of $u \in K^{r}$ we also use the Fourier transform in time only, denoted by $\hat{u}(\tau, x)=\int \mathrm{e}^{-\mathrm{i} \tau t} u(t, x) \mathrm{d} t$. For $u \in K_{0}^{r}((0, \infty) \times \mathbb{R})$ we then obtain the equivalence

$$
\|u\|_{K^{r}((0, \infty) \times \mathbb{R})}^{2} \sim \int\|\hat{u}(\tau, \cdot)\|_{H^{r}}^{2}+|\tau|^{r}\|\hat{u}(\tau, \cdot)\|_{L^{2}}^{2} \mathrm{~d} \tau .
$$

We introduce the shorthand $\mathcal{K}^{r}=K^{r} \times K^{r-1}$. Also, in this section we write $|u|_{r}$ for the Sobolev norm in the spacial variable $x$ (or its dual $k$ ), i.e., $|u|_{r}=\|u\|_{H^{r}(\mathbb{R})}$, and, e.g., $\left|\hat{u}\left(1+k^{2}\right)\right|_{0}$ for the $L^{2}$-norm of the function $k \mapsto \hat{u}\left(1+k^{2}\right)$. In the proof of Theorem 1.1 we use the following local existence theorem for the solutions of the IBLe (1.6) with $r=2$; however here we state a more general case.

Theorem A.1 Let $2 \leq r<4, \varepsilon>0$ and $t_{0}>0$ be fixed. Then there exists a $\rho>0$ such that for all $U_{0}=\left(\eta_{0}, q_{0}\right) \in H^{r}(\mathbb{R}) \times H^{r-1}(\mathbb{R})$ with $\left|U_{0}\right|_{H^{r} \times H^{r-1}} \leq \rho$ there exists a unique solution

$$
U=(\eta, q) \in \mathcal{K}^{r+1}
$$

of the IBLe (1.6) with $\left.U\right|_{t=0}=U_{0}$ and $\|U\|_{\mathcal{K}^{r+1}} \leq C\left|U_{0}\right|_{H^{r} \times H^{r-1}}$, where the constant $C>0$ depends only on $\varepsilon$ and $t_{0}$. Moreover, for all $0<t_{1}<t_{0}$ and all $k>0$ we have $U \in$ $\mathcal{K}^{r+k+1}\left(\left(t_{1}, t_{0}\right) \times \mathbb{R}\right)$, i.e., $U$ is smooth for $t>0$. 
Remark A.2 Examining the proof of Theorem A.1 we obtain that $\rho$ may be chosen independent of $\varepsilon \in\left(0, \varepsilon_{0}\right)$. Theorem A.1 is used in Corollary 3.1 in this sense, but for simplicity we do not keep track of this here. Also, the upper bound $r<4$ is only for notational convenience, i.e., to avoid the formulation of higher order trace conditions at $t=0$, see (A.12).

The proof of Theorem A.1 consists of two steps. First we consider the linear inhomogeneous version of (1.6) with zero initial data, i.e., the equation

$$
L U=F(t), \quad U(0)=0, \quad L U=U_{t}-A_{0} U, \quad F \in \mathcal{K}_{0}^{r-1},
$$

and estimate its solutions in $\mathcal{K}_{0}^{r+1}$. Then we write the solution $U$ of $(1.6)$ as $U=\tilde{U}+U^{(1)}$ where $\tilde{U} \in \mathcal{K}^{r+1}$ fulfills $\tilde{U}(0)=U_{0}$ and some (further) trace conditions at $t=0$, see (A.12). Then $U^{(1)}$ has to solve the equation

$$
L U^{(1)}=G\left(U^{(1)}\right), \quad U^{(1)}(0)=0, \quad G\left(U^{(1)}\right)=F\left(\tilde{U}+U^{(1)}\right)-L \tilde{U} .
$$

We show that for $U^{(1)} \in \mathcal{K}_{0}^{r+1}$ we have $G\left(U^{(1)}\right) \in \mathcal{K}_{0}^{r-1}$, and use the estimates for (A.4), estimates for the nonlinearity, and the contraction mapping theorem to solve (A.5).

Lemma A.3 Let $r \geq 2, \varepsilon>0$ and $t_{0}>0$. For every $F \in \mathcal{K}_{0}^{r-1}$ there exists a unique solution $U \in \mathcal{K}_{0}^{r+1}$ of (A.4) with $\|U\|_{\mathcal{K}^{r+1}} \leq C\|F\|_{\mathcal{K}^{r-1}}$, where $C>0$ depends only on $\varepsilon, t_{0}$.

Proof. We identify $F$ with its extension to $\mathcal{K}^{r-1}(\mathbb{R} \times \mathbb{R})$ with $F(t)=0$ for $t \leq 0$. Then $\mathrm{e}^{-\sigma t} F \in L^{1}\left(H^{r-1}\right) \cap L^{2}\left(H^{r-1}\right)$ for $\operatorname{Re} \sigma>0$ and therefore $\hat{F}(\tau)$ has an analytic extension into $\operatorname{Im} \tau<0$. We write $\lambda=\sigma+\mathrm{i} \tau$ and consider the Fourier transform in $t$ (i.e., the Laplace transform) of (A.4),

$$
\begin{aligned}
\lambda \hat{\eta} & =-\hat{q}_{x}+\hat{f}_{1} \Leftrightarrow \hat{\eta}=\left(-\hat{q}_{x}+\hat{f}_{1}\right) / \lambda \\
\lambda \hat{q} & =\frac{1}{\lambda}\left(a_{00}+a_{10} \partial_{x}+a_{20} \partial_{x}^{2}+\varepsilon^{-2} a_{30} \partial_{x}^{3}\right)\left(-\hat{q}_{x}+\hat{f}_{1}\right)-b_{00} \hat{q}-b_{10} \hat{q}_{x}+b_{20} \hat{q}_{x x}+\hat{f}_{2} .
\end{aligned}
$$

Now choose $\sigma_{0}>0$ such that $\operatorname{Re} \mu_{1,2}(k)<\sigma_{0}$ for all $k \in \mathbb{R}$. For $\operatorname{Re} \lambda=\sigma>\sigma_{0}$ we obtain

$$
\begin{aligned}
& |\hat{\eta}|_{r+1}+|\lambda|^{(r+1) / 2}|\hat{\eta}|_{0} \leq C\left(\left|\hat{f}_{1}\right|_{r-1}+|\lambda|^{(r-1) / 2}\left|\hat{f}_{1}\right|_{0}+\left|\hat{f}_{2}\right|_{r-2}+|\lambda|^{(r-2) / 2}\left|\hat{f}_{2}\right|_{0}\right), \\
& |\hat{q}|_{r}+|\lambda|^{r / 2}|\hat{q}|_{0} \leq C\left(\left|\hat{f}_{1}\right|_{r-1}+|\lambda|^{(r-1) / 2}\left|\hat{f}_{1}\right|_{0}+\left|\hat{f}_{2}\right|_{r-2}+|\lambda|^{(r-2) / 2}\left|\hat{f}_{2}\right|_{0}\right)
\end{aligned}
$$

see below. Moreover, since $\hat{F}$ is analytic in $\lambda$, so is $\hat{U}=(\hat{\eta}, \hat{q})$ for $\operatorname{Re} \lambda>\sigma_{0}$. Let

$$
U(t)=\frac{1}{2 \pi} \int \mathrm{e}^{\sigma_{0} t} \mathrm{e}^{\mathrm{i} \tau t} \hat{U}\left(\sigma_{0}+\mathrm{i} \tau\right) \mathrm{d} \tau .
$$

Then $\mathrm{e}^{-\sigma_{0} t} U$ is the inverse Fourier transform of the function $\tilde{\lambda} \mapsto \hat{U}\left(\sigma_{0}+\tilde{\lambda}\right)$ which is analytic for $\operatorname{Re} \tilde{\lambda}>0$. Thus, by the Paley-Wiener Theorem [23, Thm. 6.4.2] we have $U(t)=0$ for $t<0$, and from (A.2), (A.7) and (A.8) we obtain $\mathrm{e}^{-\sigma_{0} t} U \in \mathcal{K}_{0}^{r+1}\left(\mathbb{R}_{+} \times \mathbb{R}\right)$. Since $t_{0}$ is finite we thus have $U \in \mathcal{K}_{0}^{r+1}=\mathcal{K}_{0}^{r+1}\left(\left(0, t_{0}\right) \times \mathbb{R}\right)$ with $\|U\|_{\mathcal{K}^{r+1}} \leq C\|F\|_{\mathcal{K}^{r-1}}$, where $C$ obviously depends only on $t_{0}$ and $\sigma_{0}$, and hence on $t_{0}$ and $\varepsilon$. 
It remains to show (A.7),(A.8). This is essentially a direct consequence of the parabolic shape of the spectrum. After Fourier transform in $x$ and sorting terms, (A.6) becomes

$$
\hat{\hat{\eta}}=\left(-\mathrm{i} k \hat{\hat{q}}+\hat{\hat{f}}_{1}\right) / \lambda, \quad g(\lambda, k) \hat{\hat{q}}=g_{0}(k) \hat{\hat{f}}_{1}+\lambda \hat{\hat{f}}_{2},
$$

where

$$
\begin{gathered}
g(\lambda, k)=\lambda^{2}+\lambda g_{1}(k)+\mathrm{i} k g_{0}(k), \quad g_{1}(k)=b_{20} k^{2}+b_{10} \mathrm{i} k+b_{00}, \\
g_{0}(k)=a_{00}+a_{10} \mathrm{i} k-a_{20} k^{2}-a_{30} \mathrm{i} k^{3} .
\end{gathered}
$$

Since $g(\lambda, k)=\operatorname{det}\left(\lambda \mathrm{Id}-A_{0}(\mathrm{i} k)\right)=\left(\lambda-\mu_{1}(k)\right)\left(\lambda-\mu_{2}(k)\right)$, with $\mu_{1,2}$ from (1.7), we have

$$
|g(\lambda, k)| \geq C\left(|\lambda|^{2}+\left(1+k^{2}\right)^{2}\right) .
$$

Thus we can estimate

$$
\begin{gathered}
|\hat{q}|_{r} \leq C\left|\hat{\hat{q}}\left(1+k^{2}\right)^{r / 2}\right|_{0} \leq C\left(\left|\hat{\hat{f}}_{1} g_{0}(k)\left(1+k^{2}\right)^{r / 2} / g(\lambda, k)\right|_{0}+\left|\hat{\hat{f}}_{2} \lambda\left(1+k^{2}\right)^{r / 2} / g(\lambda, k)\right|_{0}\right. \\
\leq C\left(\left|\hat{\hat{f}}_{1}\left(1+k^{2}\right)^{(r-1) / 2}\right|_{0}+\left|\hat{\hat{f}}_{2}\left(1+k^{2}\right)^{(r-2) / 2}\right|_{0} \leq C\left(\left|\hat{f}_{1}\right|_{r-1}+\left|\hat{f}_{2}\right|_{r-2}\right),\right. \\
|\lambda|^{r / 2}|\hat{q}|_{0} \leq C|\lambda|^{r / 2}\left|\hat{\hat{f}}_{1} g_{0}(k) / g(\lambda, k)\right|_{0}+\left|\hat{\hat{f}}_{2} \lambda / g(\lambda, k)\right|_{0} \leq C\left(|\lambda|^{(r-2) / 2}\left(\left|\hat{\hat{f}}_{1}\right|_{0}+\left|\hat{\hat{f}}_{2}\right|_{0}\right),\right. \\
|\eta|_{r+1} \leq C\left|\left(-\mathrm{i} k \hat{\hat{q}}+\hat{\hat{f}}_{1}\right)\left(1+k^{2}\right)^{(r+1) / 2} / \lambda\right|_{0}=C\left|\frac{\left(-\mathrm{i} k g_{0}(k)+g(\lambda, k)\right) \hat{\hat{f}}_{1}-\mathrm{i} k \lambda \hat{\hat{f}}_{2}}{\lambda g(\lambda, k)}\left(1+k^{2}\right)^{(r+1) / 2}\right|_{0} \\
\quad=C\left|\frac{\left(\lambda+g_{1}(k)\right) \hat{\hat{f}}_{1}-\mathrm{i} k \hat{\hat{f}}_{2}}{g(\lambda, k)}\left(1+k^{2}\right)^{(r+1) / 2}\right|_{0} \leq C\left(\left|\hat{f}_{1}\right|_{r-1}+\left|\hat{f}_{2}\right|_{r-2}\right), \\
|\lambda|^{(r+1) / 2}|\eta|_{r+1} \leq C\left|\left(-\mathrm{i} k \hat{\hat{q}}+\hat{\hat{f}}_{1}\right) / \lambda\right|_{0} \leq C\left(|\lambda|^{(r-1) / 2}\left|\hat{\hat{f}}_{1}\right|_{0}+|\lambda|^{(r-2) / 2}\left|\hat{\hat{f}}_{2}\right|_{0}\right) .
\end{gathered}
$$

Here we used the typical parabolic splitting of the domain, for instance

$$
\begin{aligned}
\left|\frac{\hat{\hat{f}}_{2} k}{g(\lambda, k)}\right|_{0}^{2} & =\left|\frac{\hat{\hat{f}}_{2} k}{g(\lambda, k)}\right|_{L^{2}\left(\left\{k^{2} \leq|\lambda|\right\}\right)}^{2}+\left|\frac{\hat{\hat{f}}_{2} k}{g(\lambda, k)}\right|_{L^{2}\left(\left\{k^{2} \geq|\lambda|\right\}\right)}^{2}+\left.\left.|| \lambda\right|^{-3 / 2} \frac{\hat{\hat{f}}_{2} k|\lambda|^{3 / 2}}{\left(|\lambda|^{2}+\left(1+k^{2}\right)^{2}\right)}\right|_{L^{2}\left(\left\{k^{2} \geq|\lambda|\right\}\right)} ^{2} \leq C|\lambda|^{-3}\left|\hat{\hat{f}}_{2}\right|_{0}^{2} . \\
& \leq\left|\frac{k \hat{\hat{f}}_{2}}{|\lambda|^{2}}\right|_{L^{2}\left(\left\{k^{2} \leq|\lambda|\right\}\right)}^{2}
\end{aligned}
$$

The proof of Lemma A.3 is complete.

The nonlinear terms in (A.5) can be controlled using the following result, the proof of which follows via extension from $\|u v\|_{K^{s}\left(\mathbb{R} \times \mathbb{R}^{n}\right)} \leq C\|u\|_{K^{r}\left(\mathbb{R} \times \mathbb{R}^{n}\right)}\|v\|_{K^{s}\left(\mathbb{R} \times \mathbb{R}^{n}\right)}$ if $r>(n+2) / 2$.

Lemma A.4 Let $r>3 / 2,0 \leq s \leq r$. Then there exists a $C>0$ such that for all $u \in$ $K^{r}, v \in K^{s}$ we have $u v \in K^{s}$ and

$$
\|u v\|_{K^{s}} \leq C\|u\|_{K^{r}}\|v\|_{K^{s}} .
$$


Proof of Theorem A.1. Lemma A.4 applied to $F$ gives

$$
\begin{gathered}
\|F(U)\|_{\mathcal{K}^{r-1}} \leq C\|U\|_{\mathcal{K}^{r+1}}^{2} \\
\|F(U)-F(V)\|_{\mathcal{K}^{r-1}} \leq C\|U-V\|_{\mathcal{K}^{r+1}}\left(\|U\|_{\mathcal{K}^{r-1}}+\|V\|_{\mathcal{K}^{r-1}}\right)
\end{gathered}
$$

Due to [9, Thm. 4.2.3] there exists an extension $\tilde{U} \in \mathcal{K}^{r+1}$ of $U_{0} \in H^{r} \times H^{r-1}$. We have to choose $\tilde{U}=(\tilde{\eta}, \tilde{q})$ in such a way, that for $U^{(1)} \in \mathcal{K}_{0}^{r+1}$ the right hand side $G=F\left(\tilde{U}+U^{(1)}\right)-L \tilde{U}$ of (A.5) is in $\mathcal{K}_{0}^{r-1}$, i.e.,

$$
\begin{array}{ll}
\left.\partial_{t}^{k} G_{1}\right|_{t=0}=0 & \text { for } \quad 0 \leq k<r / 2-1 \\
\left.\partial_{t}^{k} G_{2}\right|_{t=0}=0 & \text { for } \quad 0 \leq k<r / 2-3 / 2
\end{array}
$$

For $r=2$ these conditions are trivially true. For $2<r \leq 3$, again due to [9, Thm. 4.2.3], we may choose $\tilde{\eta}$ in such way that $\left.\partial_{t} \tilde{\eta}\right|_{t=0}=-\partial_{x} q_{0} \in H^{r-2}(\mathbb{R})$. Similarly, for $3<r \leq 4$ we additionally choose $\tilde{q}$ such that $\left.\partial_{t} \tilde{U}\right|_{t=0}=A\left(U_{0}\right) U_{0} \in H^{r-2} \times H^{r-3}$. Thus, in each case, $L \tilde{U}=F(\tilde{U})=F\left(\tilde{U}+U^{(1)}\right)$ at $t=0$, and so $G \in \mathcal{K}_{0}^{r-1}$.

Thus, we finally consider the mapping

$$
\Phi\left(U^{(1)}\right)=L_{0}^{-1}\left(F\left(\tilde{U}+U^{(1)}\right)-L \tilde{U}\right)
$$

where $L_{0}^{-1}: \mathcal{K}_{0}^{r-1} \rightarrow \mathcal{K}_{0}^{r+1}$ is the solution operator of (A.4). If $\rho$ is sufficiently small, it is easy to see via Lemma A.3, (A.11) and the contraction mapping theorem, that $\Phi$ has a fixed point $U^{(1)}$ with $\left\|U^{(1)}\right\|_{\mathcal{K}^{r+1}} \leq C\left|U_{0}\right|_{H^{r} \times H^{r-1}}$, which gives us the solution $U=\tilde{U}+U^{(1)}$ of the IBLe.

The proof of the regularity result is standard: $U \in L^{2}\left(\left(0, t_{0}\right), H^{r+1} \times H^{r}\right)$ implies $U \in$ $H^{r+1} \times H^{r}$ for almost every $t>0$, and starting again at some such $t_{1}$ we obtain $U \in$ $\mathcal{K}^{r+2}\left(\left(t_{1}, t_{0}\right) \times \mathbb{R}\right)$. The necessary trace conditions at $t=t_{1}$ are automatically fulfilled.

Remark A.5 (A.11) holds for $r \geq 2$ due to special form of $F$, namely due to the absence of terms of the form $\eta_{x x}\left(\eta_{x x}+\eta_{x x x}\right)$ and $q_{x}\left(q_{x}+q_{x x}\right)$. If, for instance, (1.1) is expanded to cubic terms, then we obtain a term $-3 \varepsilon^{-2} \eta_{x x}^{2} \eta_{x}$ in (1.6), and then we would need $r>5 / 2$ in Theorem A.1 and therefore $m=3$ in Theorem 1.1.

\section{B Formal derivation of the IBLe}

In order to make the paper sufficiently self-contained, here we give a brief overviev of the physical problem underlying (1.1) and describe how (1.1) is formally derived.

\section{B.1 The inclined film problem}

We consider a two dimensional viscous liquid film flowing down an inclined 'plane' with inclination angle $\theta$; see figure 3 . Using $h_{0}$, the thickness of the flat film as the characteristic 


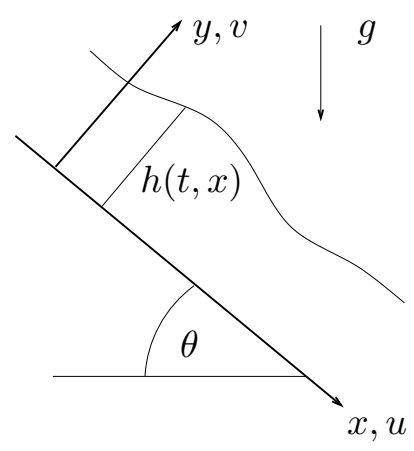

Figure 3: The inclined film problem

length and the surface velocity $u_{N}=u_{N}\left(h_{0}\right)=g h_{0}^{2} \sin \theta / 2 \nu$ of the basic Nusselt solution

$$
(u, v, p)=\left(u_{N}, 0, p_{N}\right), \quad u_{N}(y)=\frac{g \sin \theta}{2 \nu}\left(2 h_{0} y-y^{2}\right), \quad p_{N}=\rho g \cos \theta\left(h_{0}-y\right),
$$

as characteristic velocity, the governing dimensionless Navier-Stokes equations and the continuity equation read

$$
\begin{aligned}
\mathbf{u}_{t}+(\mathbf{u} \cdot \nabla) \mathbf{u} & =-\nabla p+\frac{1}{\mathrm{R}} \Delta \mathbf{u}+\frac{2}{\mathrm{R}} \mathbf{g}, \\
\operatorname{div} \mathbf{u} & =0 .
\end{aligned}
$$

Here $\mathbf{u}=(u, v)$ is the velocity field, $\mathrm{R}=u_{N} h_{0} / \nu$ is the Reynolds number, $\nu, \rho, g$ are the viscosity, density, and gravitational constant, and $\mathbf{g}=(1,-\cot \theta)$. At the free surface $y=$ $h(t, x)$ we have the kinematic condition

$$
h_{t}+h_{x} u=v,
$$

and the tangential and normal stress conditions

$$
\begin{aligned}
4 h_{x} u_{x}+\left(h_{x}^{2}-1\right)\left(u_{y}+v_{x}\right) & =0, \\
p-\frac{2}{\mathrm{R}} \frac{h_{x} u_{x}-h_{x}\left(u_{y}+v_{x}\right)+v_{y}}{1+h_{x}^{2}} & =-\mathrm{W}_{e} K(h),
\end{aligned}
$$

where $\mathrm{W}_{e}=\sigma /\left(\rho u_{N}^{2} h_{0}\right)$ is the Weber number, $\sigma$ is the coefficient of surface tension, and $K(h)=h_{x x}\left(1+h_{x}^{2}\right)^{-3 / 2}$ is the interfacial curvature. A constant athmospheric pressure $p_{a}$ has been adsorbed into $p$. Finally, at the rigid wall we prescribe the no slip condition

$$
\mathbf{u}=0 \quad \text { at } \quad y=0 .
$$

In dimensionless variables the Nusselt solution is

$$
(u, v, p, h)(t, x, y)=\left(u_{N}, 0, p_{N}, 1\right), \quad u_{N}=2 y-y^{2}, \quad p_{N}(y)=2 \cot \theta(1-y) / \mathrm{R},
$$


and from previous work [2] it is well known that it is unstable to long wave perturbations for Reynolds numbers

$$
\mathrm{R}>\mathrm{R}_{c}=\frac{5}{4} \cot \theta .
$$

In order to analyze this long wave instability, a number of reduced equations for (B.1) as for instance the so called Boundary Layer equation, the Integral Boundary Layer equation, and the Kuramoto-Sivashinsky equation have been derived. We now briefly describe the derivation of the IBLe for (B.1). For notational convenience we consider directly (B.1), rather than shifting the origin to $\left(u_{N}, 0, p_{N}, 1\right)$. The Nusselt solution will thus appear in the perturbation analysis as a zeroth order approximation.

\section{B.2 Derivation of the IBLe}

We assume that the Weber number is large, $\mathrm{W}_{e}=\mathrm{W} \varepsilon^{-2}$ where $0<\varepsilon \ll 1$ is a small parameter, while the Reynolds number is $\mathcal{O}(1)$, and let

$$
\begin{aligned}
& u(t, x, y)=\tilde{u}(\tau, \xi, y), \quad v(t, x, y)=\varepsilon^{2 / 3} \tilde{v}(\tau, \xi, y), \quad \tau=\varepsilon^{2 / 3} t, \quad \xi=\varepsilon^{2 / 3} x, \\
& p(t, x, y)=\varepsilon^{-2 / 3} \tilde{p}(\tau, \xi, y), \quad h(t, x)=\tilde{h}(\tau, \xi) .
\end{aligned}
$$

Substituting this long wave ansatz into the free boundary value problem (B.1) and retaining terms up to order $\mathcal{O}\left(\varepsilon^{4 / 3}\right)$ we obtain

$$
\begin{aligned}
\operatorname{in} \Omega: \quad & \varepsilon^{2 / 3}\left(\tilde{u}_{\tau}+\tilde{u}_{\xi} \tilde{u}+\tilde{u}_{y} \tilde{v}\right)=-\tilde{p}_{\xi}+\left(\varepsilon^{4 / 3} \tilde{u}_{\xi \xi}+\tilde{u}_{y y}+2\right) / \mathrm{R}, \\
& 0=-\tilde{p}_{y}-2 \varepsilon^{2 / 3} \cot \theta / \mathrm{R}+\varepsilon^{4 / 3} \tilde{v}_{y y} / \mathrm{R}, \\
& \tilde{u}_{\xi}=-\tilde{v}_{y} \\
\text { at } y=\tilde{h}(t, \xi): \quad & \tilde{h}_{\tau}+\tilde{h}_{\xi} \tilde{u}=\tilde{v}, \\
& -\tilde{u}_{y}+\varepsilon^{4 / 3}\left(\tilde{h}_{\xi} \tilde{u}_{\xi}+\tilde{h}_{\xi}^{2} \tilde{u}_{y}-\tilde{v}_{\xi}\right)=0, \\
& \tilde{p}-2 \varepsilon^{4 / 3}\left(-\tilde{u}_{\xi}-\tilde{h}_{\xi} \tilde{u}_{y}\right) / \mathrm{R}=-\mathrm{W} \tilde{h}_{\xi \xi}\left(1-(3 / 2) \varepsilon^{4 / 3} \tilde{h}_{\xi}^{2}\right), \\
\text { at } y=0: \quad & \tilde{u}=\tilde{v}=0 .
\end{aligned}
$$

In order to derive the Integral Boundary Layer equation we define the flow rate

$$
\tilde{q}(\tau, \xi)=\int_{0}^{\tilde{h}(\tau, \xi)} \tilde{u}(\xi, y) \mathrm{d} y .
$$

Then (B.3a), (B.3d) are equivalent to

$$
\begin{aligned}
\tilde{h}_{\tau}= & -q_{\xi}, \\
\tilde{q}_{\tau}= & -\partial_{\xi} \int_{0}^{\tilde{h}} \tilde{u}^{2} \mathrm{~d} y-\varepsilon^{-2 / 3} \int_{0}^{\tilde{h}} \tilde{p}_{\xi} \mathrm{d} y \\
& +\varepsilon^{-2 / 3}\left[\varepsilon^{4 / 3} \int_{0}^{\tilde{h}} \tilde{u}_{\xi \xi} \mathrm{d} y+\left(\tilde{u}_{y}(y=\tilde{h})-\tilde{u}_{y}(y=0)\right)+2 \tilde{h}\right] / \mathrm{R} .
\end{aligned}
$$


Following [20], we assume that the velocity field is slaved to the elevation $\tilde{h}$ and the flow $\tilde{q}$ in a Nusselt like fashion, i.e.,

$$
\tilde{u}=\frac{3 \tilde{q}}{2 \tilde{h}^{3}}\left(2 \tilde{h} y-y^{2}\right)
$$

From (B.3f), (B.3b) and (B.3c) we may integrate the so called hydrostatic head, i.e., we calculate

$$
\begin{aligned}
\tilde{p}(y) & =\tilde{p}(\tilde{h})-\int_{y}^{\tilde{h}} \tilde{p}_{y} \mathrm{~d} y=\tilde{p}(\tilde{h})+\varepsilon^{2 / 3} \int_{y}^{\tilde{h}} 2 \cot \theta / \mathrm{R}+\varepsilon^{2 / 3} \tilde{u}_{x y} \mathrm{~d} y \\
& =-\mathrm{W} \tilde{h}_{\xi \xi}\left(1-(3 / 2) \varepsilon^{4 / 3} \tilde{h}_{\xi}^{2}\right)+\frac{1}{\mathrm{R}}\left(2 \varepsilon^{2 / 3} \cot \theta(\tilde{h}-y)-\varepsilon^{4 / 3}\left(\tilde{u}_{\xi}(y=\tilde{h})+\tilde{u}_{\xi}(y)\right)\right) .
\end{aligned}
$$

Substituting this and (B.5) into (B.4) we obtain

$$
\begin{aligned}
\tilde{q}_{\tau}= & -\frac{6}{5} \partial_{\xi}\left(\tilde{q}^{2} / \tilde{h}\right)+\varepsilon^{-2 / 3}\left[\tilde{h} \mathrm{~W}\left(\tilde{h}_{\xi \xi \xi}\left(1-3 \varepsilon^{4 / 3} \tilde{h}_{\xi}^{2} / 2\right)-3 \varepsilon^{4 / 3} \tilde{h}_{\xi \xi}^{2} \tilde{h}_{\xi}\right)+\left(2 \tilde{h}-3 \tilde{q} / \tilde{h}^{2}\right) / \mathrm{R}\right] \\
& -2 \cot \theta \tilde{h}_{\xi} \tilde{h} / \mathrm{R}+\frac{\varepsilon^{2 / 3}}{\mathrm{R}}\left[(7 / 2) \tilde{q}_{\xi \xi}-9 \tilde{q}_{\xi} \tilde{h}_{\xi} / \tilde{h}+6 \tilde{q} \tilde{h}_{\xi}^{2} / \tilde{h}^{2}-9 \tilde{q} \tilde{h}_{\xi \xi} /(2 \tilde{h})\right] .
\end{aligned}
$$

Finally, (B.4) becomes (1.1) when scaling back to $t, x$, i.e., defining

$$
h(t, x)=\tilde{h}\left(\varepsilon^{-2 / 3} \tau, \varepsilon^{-2 / 3} \xi\right), \quad q(t, x)=\tilde{q}\left(\varepsilon^{-2 / 3} \tau, \varepsilon^{-2 / 3} \xi\right) .
$$

Remark B.1 Evaluating the assumption (B.6) mathematically seems rather difficult. Note that with this assumption, and defining $\tilde{v}(\tau, \xi, y)=-\int_{0}^{y} \tilde{u}_{\xi}(\tau, \xi, \tilde{y}) \mathrm{d} \tilde{y}$ the no slip boundary condition (B.3g) is fulfilled, but the condition (B.3e) for the tangential stress only up to order $\mathcal{O}\left(\varepsilon^{4 / 3}\right)$. See also the following subsection.

\section{B.3 Remarks on first order Boundary Layer Theory}

If in (B.3) we keep terms only up to order $\mathcal{O}\left(\varepsilon^{2 / 3}\right)$ we obtain the so called Boundary Layer equation [5]: the hydrostatic head gives $\tilde{p}(y)=-\mathrm{W} \tilde{h}_{\xi \xi}+\varepsilon^{2 / 3} \cot \theta(\tilde{h}-y) / \mathrm{R}$, and thus

$$
\begin{aligned}
\operatorname{in} \Omega: & \tilde{u}_{\tau}+\tilde{u}_{\xi} \tilde{u}+\tilde{u}_{y} \tilde{u}=\frac{\varepsilon^{-2 / 3}}{\mathrm{R}}\left[\tilde{u}_{y y}+2+\mathrm{RW} \tilde{h}_{\xi \xi \xi}\right]-2 \cot \theta \tilde{h}_{\xi} / \mathrm{R}, \\
& \tilde{u}_{\xi}=-\tilde{v}_{y}, \\
\text { at } y=\tilde{h}(t, \xi): & \tilde{h}_{\tau}+\tilde{h}_{\xi} \tilde{u}=\tilde{v}, \quad \tilde{u}_{y}=0, \\
\text { at } y=0: \quad & \tilde{u}=\tilde{v}=0 .
\end{aligned}
$$

In this case, the ansatz (B.5) gives

$$
\begin{aligned}
\tilde{h}_{\tau} & =-\tilde{q}_{\xi} \\
\tilde{q}_{\tau} & =-\frac{6}{5} \partial_{\xi}\left(\tilde{q}^{2} / \tilde{h}\right)+\frac{\varepsilon^{-2 / 3}}{\mathrm{R}}\left[2 \tilde{h}-3 \tilde{q} / \tilde{h}^{2}+\mathrm{RW} \tilde{h} \partial_{\xi}^{3} \tilde{h}\right]-2 \cot \theta \tilde{h}_{\xi} \tilde{h} / \mathrm{R},
\end{aligned}
$$


and the reduction of (B.7) to (B.8) is exact, i.e., every solution of (B.8) gives an exact solution of (B.7) via (B.5). In other words, the solutions of (B.8) define an invariant manifold for (B.7). Moreover, (B.8) reduces to the Kuramoto-Sivashinsky equation (1.14) in just the same way as (1.1) does, since the dissipation terms first show up in the $q$-equation at order $\mathcal{O}\left(\varepsilon^{3}\right)$.

However, (B.8) is a quasilinear hyperbolic equation, as can be seen from the dispersion relation

$$
\mu_{1,2}(k)=-\frac{1}{2}\left(\frac{3}{\mathrm{R}}+\frac{8}{5} \mathrm{i} k\right) \pm \sqrt{\frac{1}{4}\left(\frac{3}{\mathrm{R}}+\frac{8}{5} \mathrm{i} k\right)^{2}-\frac{6}{\mathrm{R}} \mathrm{i} k-\left(\frac{4}{5}-\frac{2}{\mathrm{R}} \cot \theta\right) k^{2}-\mathrm{W} \varepsilon^{-2} k^{4}}
$$

for the linearization of (B.8) (after rescaling to $t, x$ coordinates) around $(q, h)=(2 / 3,1)$. Therefore, with our method we can not prove an approximation result like Theorem 1.1 for the reduction of (B.8) to the Kuramoto-Sivashinsky equation. The reason is, that due to the lack of dissipation the energy estimate for the quasilinear problem breaks down.

\section{References}

[1] J.T. Beale. Large time regularity of viscous surface waves. Arch. Rat. Mech. Anal., 84:307-352, 1984

[2] T.B. Benjamin. Wave formation in laminar flow down an inclined plane. J. Fluid Mech., 2:554-574, 1957.

[3] H.-C. Chang and E. A. Demekhin. Solitary wave formation and dynamics on falling films. Adv. Appl. Mech., 32:1-58, 1996.

[4] H.-C. Chang, E.A. Demekhin, and E. Kalaidin. Generation and suppression of radiation by solitary pulses. SIAM J. Appl. Math., 58(4):1246-1277, 1998.

[5] H.-C. Chang, E.A. Demekhin, and D. I. Kopelevich. Nonlinear evolution of waves on a vertically falling film. J. Fluid Mech., 250:433-480, 1993.

[6] H.-C. Chang, E.A. Demekhin, and D.I. Kopelevich. Local stability theory of solitary pulses in an active medium. Physica D, 97:353-375, 1996.

[7] Th. Gallay and G. Schneider. KP-description of unidirectional dispersive waves - The model case. Proc. Royal Soc. Edinb. A, 131(4):885-898, 2001.

[8] P. Kirrmann, G. Schneider, and A. Mielke. The validity of modulation equations for extended systems with cubic nonlinearities. Proc. of the Royal Society of Edinburgh, 122A:85-91, 1992.

[9] J.L. Lions and E. Magenes. Problèmes aux limites non homogènes. Dunod, Paris, 1968.

[10] J. Liu and J.P. Gollub. Solitary wave dynamics of film flows. Phys. Fluids, 6(5):17021712, 1994. 
[11] P. Manneville. The Kuramoto-Sivashinky equation: a progress report. In J.E. Wesfreid et al, editor, Propagation in systems far from equilibrium (Les Houches, 1987), pages 265-280. Springer Ser. Synergetics, 41, Springer, Berlin, 1988.

[12] A. Mielke and G. Schneider. Attractors for modulation equations on unbounded domains-existence and comparison. Nonlinearity, 8(5):743-768, 1995.

[13] Toshiyuki Ogawa and Hiromasa Susuki. On the spectra of pulses in a nearly integrable system. SIAM J. Appl. Math., 57(2):485-500, 1997.

[14] B. Ramaswamy, S. Chippada, and S. W. Joo. A full scale numerical study of interfacial instabilities in thin film flows. J. Fluid Mech., 325:163-194, 1996.

[15] G. Schneider. Error estimates for the Ginzburg-Landau approximation. ZAMP, 45:433457, 1994.

[16] G. Schneider. A new estimate for the Ginzburg-Landau approximation on the real axis. J. Nonlinear Science, 4:23-34, 1994.

[17] G. Schneider. Validity and Limitation of the Newell-Whitehead equation. Math. Nachr., 176:249-263, 1995.

[18] G. Schneider. Global existence results in pattern forming systems - Applications to 3D Navier-Stokes problems -. J. Math. Pures Appl., IX, 78:265-312, 1999.

[19] G. Schneider and C.E. Wayne. The long wave limit for the water wave problem I. The case of zero surface tension. Comm. Pure. Appl. Math., 53(12):1475-1535, 2000.

[20] W.Ya. Shkadov. Wave conditions in the flow of a thin layer of a viscous liquid under the action of gravity. Izv. Akad. Nauk SSSR, Mekh. Zhidk. Gaza, 1:43-50, 1967.

[21] Y. Teramoto. On the Navier-Stokes flow down an inclined plane. J. Math. Kyoto Univ., 32(3):593-619, 1992.

[22] J. Topper and T. Kawahara. Approximate equations for long nonlinear waves on a viscous fluid. J. Phys. Soc. Japan, 44(2):663-666, 1978.

[23] K. Yosida. Functional Analysis. Springer, New York, 1971. 